\title{
Further studies of pigeons' spatial working memory in the open-field task
}

\author{
MARCIA L. SPETCH \\ University of Alberta, Edmonton, Alberta, Canada
}

\begin{abstract}
In Experiments 1 and 2, pigeons' spatial working memory in an open-field setting was examined under conditions that differed in terms of working-memory load (number of sites visited prior to a retention test) at various delays between initial choices and the retention test. In Experiment 1, pigeons were tested under two conditions of memory load (three or five sites visited prior to the delay) and two delay intervals (15 and $60 \mathrm{~min}$ ). Accuracy declined as a function of delay but was not affected significantly by memory load. In Experiment 2A, pigeons were tested under three conditions of memory load (two, four, or six sites visited prior to the delay). In separate phases, the delay was 2,15 , and $60 \mathrm{~min}$. Accuracy was not affected by memory load in any of these phases. In Experiment 2B, three conditions of memory load (two, four, or six sites visited prior to the delay) were tested at two delays ( 2 and $60 \mathrm{~min}$ ) within a test phase. Accuracy declined with increasing delay, but memory load again had no significant effects. These results are inconsistent with previous suggestions that pigeons' retention of spatial information may decline as working-memory load is increased. In Experiment 3, cue-manipulation tests confirmed that pigeons' choice behavior in the open-field task is controlled by memory for previously visited room locations.
\end{abstract}

Initial investigations had suggested that pigeons may not share rats' ability to accurately remember which arms of a radial maze they have already visited (Bond, Cook, \& Lamb, 1981). However, subsequent work has revealed that, with special or extended training, pigeons can exhibit very accurate spatial working memory in radial-arm mazes (Dale, 1988; Roberts \& Van Veldhuizen, 1985). Moreover, pigeons will readily display accurate spatial working memory in a T-maze (Olson \& Maki, 1983) or in an open-field setting that contains eight feeding stations (Spetch \& Edwards, 1986; Spetch \& Honig, 1988). Thus, pigeons are capable of remembering locations they have recently visited, but their readiness to base choice responses on this memory appears to depend in part on the structure of the test apparatus.

Apart from special training and/or apparatus requirements, pigeons' spatial working memory seems to be similar to that of rats in several respects. For example, pigeons, like rats, appear to preferentially use extramaze cues in radial maze tasks (Dale, 1988). Pigeons also show proactive interference effects with massed trials (Roberts \& Van Veldhuizen, 1985) similar to such effects shown by rats (Roberts \& Dale, 1981).

Pigeons' spatial working memory may, however, differ from that of rats in terms of durability. Pigeons' accuracy in an eight-arm maze has been found to decline signifi-

This research was supported by a Natural Sciences and Engineering Research Council of Canada operating grant (OGP0038861). I wish to thank E. Buckle and M. Fundytus for their assistance in testing the animals, and Doug Grant, Dallas Treit, and two anonymous reviewers for their helpful comments on an earlier version of this manuscript. Requests of reprints should be addressed to M. Spetch, Department of Psychology, University of Alberta, Edmonton, Alberta T6G 2E9, Canada. cantly over delay intervals of only $6 \mathrm{~min}$ (Roberts \& Van Veldhuizen, 1985). Recently, Spetch and Honig (1988) found that pigeons were able to maintain accurate performance in an eight-choice open-field task for delays of up to $32 \mathrm{~min}$. However, even this value does not compare favorably with the 4-h delays over which rats have been found to perform in an eight-arm maze without significant memory loss.

It has been suggested (Roberts \& Van Veldhuizen, 1985) that the maximal delay over which pigeons can accurately remember spatial locations without significant loss may be reduced by increments in memory load. This suggestion was based on a comparison of Roberts and Van Veldhuizen's (1985) finding that pigeons given four forced choices in an eight-arm maze showed significant forgetting over a 6-min delay interval, with Olson and Maki's (1983) finding that pigeons were able to maintain accurate memory of a single arm of a T-maze over a 16min delay. Roberts and Van Veldhuizen suggested that the increase in memory load from one spatial location in the T-maze to four spatial locations in the eight-arm maze may have promoted forgetting. Further indirect support for this notion was subsequently provided by Dale (1988). He tested pigeons on a four-arm maze and found that the delay over which they could maintain accurate performance was intermediate to that found by Roberts and Van Veldhuizen for the eight-arm maze and the delay found by Olson and Maki for the two-alley T-maze. Roberts \& Van Veldhuizen also tentatively suggested that retention in pigeons might be more susceptible to loss through an increase in memory load than is retention in rats.

Although provocative, the suggested relationship between memory load and retention in pigeons has been 
based only on indirect evidence provided by betweenexperiment comparisons. Furthermore, even if direct within-experiment comparisons of pigeons' performance on a two-arm and an eight-arm maze confirmed the suggested differences in retention, the specific factor underlying this difference would need to be clarified. There are at least three differences among the retention tests conducted with the T-maze (Olson \& Maki, 1983), the fourarm maze (Dale, 1988), and the eight-arm maze (Roberts \& Van Veldhuizen, 1985). First, in the T-maze, the pigeons visited only one arm before the delay, whereas in the four-arm maze they visited three arms before the delay, and in the eight-arm maze they visited four arms. Thus, the tests with the T-maze entailed a "workingmemory" load of only one arm, whereas those in the fourarm and eight-arm mazes entailed working-memory loads of three and four arms, respectively. Second, in the Tmaze, there were only two arms in the total "referencememory set," compared to four in the four-arm maze and eight in the eight-arm maze (see Honig, 1978, for a general discussion of the distinction between working and reference memory). Finally, the number of arms available during the choice test that followed the delay was different (two in the T-maze, four in the four-arm maze, and eight in the eight-arm maze).

Experiments 1 and 2 were designed to examine the effect of working-memory load on pigeons' retention ability, while holding these other factors constant. Pigeons were trained in an open-field spatial working-memory task with a constant reference-memory set of eight food sites. The number of food sites available for choice following the delay was also held constant (six in Experiment 1, four in Experiments 2A and 2B), but the number of sites visited prior to the delay (i.e., working-memory load) was varied. Retention accuracy was examined at two values of workingmemory load and at two delays in Experiment 1, at three values of working-memory load and three delays in Experiment $2 \mathrm{~A}$, and at three values of working-memory load and two delays in Experiment 2B. Of particular interest was whether the retention functions would be steeper under conditions of higher working-memory load.

Experiment 3 focused on the cues that control pigeons' working memory in the open-field task. In Experiments 1 and 2, various local and distal cues were present that could have been used by the pigeons to identify the visited sites. In Experiment 3, some of these cues were manipulated during the delay period to determine their relative importance.

\section{EXPERIMENT 1}

In this experiment, pigeons trained in the open-field setting with eight food sites were given forced-choice tests in which the working-memory load and the delay interval were varied across trials. During the sample portion of the trial, the pigeons visited either three or five randomly selected sites. After a delay of either 15 or $60 \mathrm{~min}$, they were tested with six sites, three of which were correct (i.e., not visited on the current trial) and three of which were incorrect. Of interest was whether the pigeons would make more errors when they had been to five sites prior to the delay than when they had been to only three sites.

\section{Method}

Subjects. The subjects were 4 adult White King pigeons that had previously been trained and tested in an open-field spatial memory task (Spetch \& Honig, 1988). They were maintained at approximately $85 \%$ of their free-feeding weights by food given during and after the experimental sessions. The pigeons were housed in individual wire-mesh cages with water and grit freely available.

Apparatus. The open-field arena consisted of a rectangular test room, $2.4 \times 2.3 \mathrm{~m}$, with a door on one wall and two windows on the other. One window contained one-way glass through which the test room could be viewed from a separate observation room; the other window contained frosted glass. The four inside walls of the test room were white, but contained some distinctive features (e.g., sockets, doorstop, light switch, the windows, the door, etc.) that could serve as spatial landmarks. In addition to these static landmarks, the room contained four surface landmarks (a rock, a long stick, a block of wood, and an inverted plastic bowl) and three distinct wall landmarks (photographs of a swan, a flower, and a sunset, mounted on backgrounds of different sizes, colors, and shapes). Illumination was provided by a single overhead lightbulb.

The eight food sites were each constructed of 2-liter milk cartons that differed in color and patterning. The cartons were modified so that they were open at the top with a semicircular entrance hole on the front side. An additional strip of cardboard (of the same visual design as the carton) was attached to the top to provide extra height and "flaps" to border the entrance. The cartons were filled with grit to $10 \mathrm{~cm}$ below the entrance, and they were baited by placing kernels of corn on the grit against the front wall of the carton, beneath the entrance. Each carton was placed on a wooden platform. A ramp, which served as a walkway to the carton entrance, was attached to the front of each platform; strips of wood were glued onto the ramp to provide rungs. The eight cartons were spaced approximately $0.4 \mathrm{~m}$ apart and were arranged in an irregular circle with the ramps facing the center (see Spetch \& Honig, 1988).

Procedure. The pigeons' behavior was observed through oneway glass by an experimenter located in the observation room. Site choices (visits) were operationally defined as the bird stepping with both feet onto the ramp that led to a food carton. Previously conducted control tests had indicated that the pigeons could not see the food hidden inside the cartons until they had walked up the ramp to the carton entrance. The experimenter recorded the order of each choice response.

Since all birds had been trained on a spatial working-memory task, no preliminary training was necessary. The basic procedure used for this experiment was as follows. During the sample portion of each trial, the pigeons received a forced choice of either three (FC-3) or five (FC-5) randomly selected food sites. These sample sites were each baited with six kernels of corn and placed in their designated locations in the room. The other sites were removed from their locations, but with the ramps left in place. The pigeon was placed in the center of the room with the light out. Once the experimenter arrived at the observation window, the test-room light was turned on and the pigeon was free to choose among the available sites until it had visited each one. The light was then turned out and the bird was removed and placed in a holding cage in a darkened adjacent room for a delay of either 15 or $60 \mathrm{~min}$. During the retention test that followed the delay, the room contained three previously visited sites and three sites that had not been available during the sample portion of the trial; only the latter, unvisited sites were baited with food. The pigeon was permitted to choose among these six sites until it had visited each of the three baited sites. 


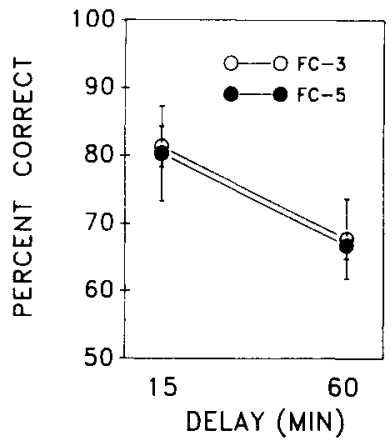

Figure 1. Mean retention accuracy as a function of delay for the FC- 3 condition (three sites visited prior to the delay) and the FC-5 condition (five sites visited prior to the delay) in Experiment 1. Vertical bars indicate standard error of the mean. Chance level is $50 \%$.

The four trial types (FC-3 and FC-5 with a 15-min delay, FC-3 and FC-5 with a 60-min delay) each occurred once in every fourtrial block, but in a mixed order. Testing continued until each type of trial had occurred eight times. Trials were conducted once per day, 5 days per week.

Analysis of choice accuracy. Because the focus of interest was on how well the birds remembered which sites they had visited prior to the delay, the primary measure of choice accuracy was the number correct out of the first three different sites chosen during the retention test (see Spetch \& Honig, 1988). This measure, referred to as retention accuracy, excludes any repeat errors during the test, and has a chance-level accuracy of 1.5 sites (i.e., $50 \%$ correct choices). In this experiment, no repeat errors were made during the retention test. Retention-accuracy scores were transformed to percent-correct scores for graphic presentation.

\section{Results}

Figure 1 shows retention accuracy as a function of delay for the two conditions of working-memory load. Although accuracy declined as a function of delay, the larger memory-load condition (FC-5) did not generate a steeper function.

A two-way repeated measures analysis of variance confirmed that there was a significant main effect of delay $[F(1,3)=20.75, p<.05]$, but not of memory load $[F(1,3)<1.0]$, and there was no significant delay $\times$ memory load interaction $[F(1,3)<1.0]$. Retention accuracy was significantly above chance level $(50 \%$ correct) at both the $15-\min$ delay $[t(3)=7.36, p<.01]$ and the 60 -min delay $[t(3)=6.52, p<.01]$.

Repeated visits to a site during the sample portion of the trial could affect retention accuracy by effectively increasing sample exposure. Therefore, to eliminate the contribution of any increased sample exposure produced by repeat errors, all trials containing a repeated sample choice were excluded, and the data were reanalyzed. This analysis did not change the pattern of results: there was a significant effect of delay $[F(1,3)=12.05, p<.05]$, but no other significant effects.

Table 1 shows the retention-accuracy scores for the individual subjects. The scores obtained after excluding trials with repeated choices of a sample are also shown.

\section{Discussion}

The pigeons' accuracy in selecting the new sites over ones they had already visited within the current trial did not depend on whether they had visited three or five different sites prior to the delay. Although this would suggest that working-memory load may not affect pigeons' ability to remember which sites they have visited, it is possible that the memory-load range (3-5) used in this experiment was too narrow.

Alternatively, it is possible that the two conditions used in this experiment actually represented equal conditions of working-memory load (Cook, Brown, \& Riley, 1985). That is, in spatial working-me,nory tasks, animals might use information about previously visited sites (retrospective memory) during their initial choices, but later in the trial they might use information about anticipated choices (prospective memory). During the course of a trial, the number of visited sites (retrospective memory load) increases, whereas the number of anticipated site choices (prospective memory load) decreases. With a flexible use of retrospective or prospective information, the largest memory load would be in effect when the subjects had visited half of the sites in the total set. For example, with eight sites in the set, visiting three sites prior to the delay would entail a retrospective memory load of three, and visiting five sites would entail a prospective memory load of three. However, visiting four sites would entail a memory load of four with both retrospective and prospective memories.

Evidence suggesting that rats may be able to use either retrospective or prospective information for spatial memory has been provided by Cook et al. (1985). They found that rats performed less accurately if a 15 -min delay followed a forced choice to 6 of the arms of a 12-arm maze than if it followed a forced choice to either fewer or more than six of the arms. However, this effect interacted with delay length: a quadratic function relating accuracy to the number of arms visited prior to the delay was obtained with a 15 -min delay, but not with a 60 -min delay.

A recent study by Zentall, Steirn, and Jackson-Smith (1987), using an operant analogue of the radial-arm maze,

Table 1

Retention-Accuracy Scores for the 4 Subjects in Experiment 1

\begin{tabular}{|c|c|c|c|c|c|c|c|c|}
\hline \multirow[b]{4}{*}{ Subject } & \multicolumn{8}{|c|}{ Delay } \\
\hline & \multicolumn{4}{|c|}{$15 \mathrm{~min}$} & \multicolumn{4}{|c|}{$60 \mathrm{~min}$} \\
\hline & \multicolumn{2}{|c|}{ FC-3 } & \multicolumn{2}{|c|}{ FC-5 } & \multicolumn{2}{|c|}{ FC-3 } & \multicolumn{2}{|c|}{ FC-5 } \\
\hline & RE & NRE & RE & NRE & RE & NRE & RE & NRE \\
\hline 218 & 2.38 & 2.38 & 2.75 & 2.75 & 2.12 & 2.29 & 1.88 & 1.88 \\
\hline 3241 & 2.50 & 2.50 & 2.38 & 2.38 & 2.38 & 2.38 & 2.00 & 2.00 \\
\hline 219 & 2.62 & 2.62 & 2.62 & 2.67 & 2.00 & 2.00 & 2.12 & 2.14 \\
\hline 440 & 2.25 & 2.33 & 1.88 & 1.67 & 1.62 & 1.57 & 2.00 & 2.17 \\
\hline
\end{tabular}

Note-Data represent the number correct out of three choices. FC-3 $=$ forced choice of three randomly selected sites, FC-5 $=$ forced choice of five randomly selected sites, $\mathrm{RE}=$ retention-accuracy scores with repeat errors during the sample phase included, NRE = retentionaccuracy scores with repeat errors during the sample phase excluded. 
produced similar results with pigeons. In their study, pigeons were presented with an array of five pecking keys, and they received food only for the first choice of a given key on each trial. Delays were inserted selectively between choices, and the results showed an inverted U-shaped function: the birds were more accurate if the delay occurred after they had selected one or four of the keys than if it occurred after they had selected two or three of the keys.

In view of these results, it seems possible that memoryload effects were not observed in the present experiment because the FC- 3 and FC-5 trials failed to provide functionally different memory loads.

\section{EXPERIMENT 2}

Given the possibility that pigeons might use retrospective and prospective information at different points within a trial, it seemed worthwhile to replicate the first experiment, but with the inclusion of an intermediate memoryload condition. Therefore, in Experiment 2, pigeons visited two, four, or six sites during the sample portion of the trial. During the choice test that followed a delay, four sites were available, two correct and two incorrect. In Experiment $2 \mathrm{~A}$, memory load was assessed at three delay intervals $(2,15$, and $60 \mathrm{~min})$ in separate experimental phases. In Experiment $2 B$, the three conditions of memory load were assessed at two delay intervals ( 2 and $60 \mathrm{~min}$ ) within a test phase.

\section{Experiment 2A}

\section{Method}

Subjects. The subjects were 4 experimentally naive Silver King pigeons, approximately 1 year of age. They were maintained at $85 \%$ of their free-feeding weights by corn given during the experimental sessions and supplemental feedings of mixed grain. The subjects were housed in individual wire-mesh cages, under a 12:12 h dark: light cycle. Water and grit were freely available in the home cages.

Apparatus. The test room was rectangular and was approximately $3.3 \mathrm{~m}$ long and $2.9 \mathrm{~m}$ wide. One wall contained the door, and the other three walls each contained a window. Two of the windows were covered with black plastic; the third window contained oneway glass and served as the observation window for viewing the birds from an adjacent room. One corner of the test room contained a plywood enclosure with a steeply sloped top that covered and prevented access to a sink. Other features of the room included the light switch and wall sockets, four scenery pictures of different sizes and shapes that were placed on the walls, and four objects (a rock, a stick, artificial potted flowers, and a $\log$ ) that were placed on the floor of the room. Illumination was provided by four fluorescent overhead lights that could be controlled from the adjacent observation room. Background masking noise was provided by a radio tuned off station.

The food sites consisted of eight cartons and ramps that were similar to those used in Experiment 1 . The sites were arranged in a circle that was approximately $1.7 \mathrm{~m}$ in diameter, with the ramps pointing toward the center. The sites were spaced approximately $0.6 \mathrm{~m}$ apart, measured from the tips of the ramps.

Procedure. The birds were always placed in and removed from the test room with the overhead lights out. The experimenter turned on the test-room lights from within the observation room to start a trial, and turned them off to end it. The experimenter observed the pigeon through one-way glass and recorded the order of each choice of a site, defined in terms of a bird stepping onto the ramp with both feet.

Each bird received between 4 and 1030 -min pretraining trials in which it was adapted to the test room and shaped to locate and eat food from the cartons. Initially, only one carton was placed in the room and food was located on the ramp as well as inside the carton. Once the pigeon readily ate from this carton, the other food sites were gradually introduced. Once the bird ate from all cartons, food was no longer placed on the ramps. Pretraining continued until the bird located and ate the food from all eight cartons within $15 \mathrm{~min}$.

Following pretraining, each bird received 10 free-choice trials with all eight food sites. The bird was placed in the room with all sites baited and was allowed to choose until it had visited all eight sites, or for a maximum of $15 \mathrm{~min}$.

The memory-load tests used a forced-choice procedure and entailed three phases. During Phase 1, the delay between the forced choice and the test part of the trial was 2 min; during Phase 2, it was $15 \mathrm{~min}$; and during Phase 3, it was $60 \mathrm{~min}$. Each phase lasted for 30 trials, and the procedure during each phase was identical except for the delay interval. Within each phase, three conditions of memory load-FC-2, FC-4, and FC-6-each occurred once during each three-trial block, but in a randomly determined order.

On FC-2 trials, two randomly selected sites were available during the sample part of the trial: the bird was placed in the room with only these sites in place (all other cartons and ramps were removed from the room). Once the bird ate from both sites, it was removed from the room and placed in a holding cage in a separate darkened room while two additional randomly selected sites were baited and placed in the room. Following the specified delay interval, the bird was returned to the test room and was allowed to choose among the four sites until it had eaten from the two baited ones. The procedure was the same on FC -4 and FC- 6 trials, except that the number of sites available during the sample period was four and six, respectively. Two of these sites, together with two of the remaining sites, were randomly selected to be available during the retention test.

Throughout all phases of the experiment, the pigeons were given a single trial each day, and these occurred 5 or 6 days per week. A 10-min time limit was imposed for both the sample and the test portions of each trial. If the bird failed to visit each of the sample sites within $10 \mathrm{~min}$, or if it failed to make at least two choices within $10 \mathrm{~min}$ of the retention test, the trial was aborted. Across the three test phases, this occurred on 6 out of $120 \mathrm{FC}-2$ trials, on 8 out of 120 FC -4 trials, and on 7 out of 120 FC -6 trials.

Analysis of choice accuracy. During free choice, accuracy was determined by the number of different sites chosen during the first eight choices. During the forced-choice tests, the retention-accuracy measure used in Experiment 1 was again employed. With the present design, chance level for retention accuracy (number correct out of the first two different sites chosen) is 1.0 sites, or $50 \%$ correct. Repeat errors during the first two choices of the retention test occurred only very occasionally, and an analysis that included these errors yielded mean accuracy scores that did not differ from the scores reported by more than $4 \%$ in any of the conditions. Moreover, the pattern of significant and nonsignificant effects remained unchanged.

\section{Results}

Free-choice performance. Averaged over the last five trials, accuracy during the free-choice phase was $6.4,6.4$, 7.0, and 6.4 correct choices out of 8 for Pigeons 73, 539, 37 , and $\mathbf{S 8 4}$, respectively. These scores are well above the level expected on the basis of random sampling among the eight alternatives (5.6), although they are not as high 


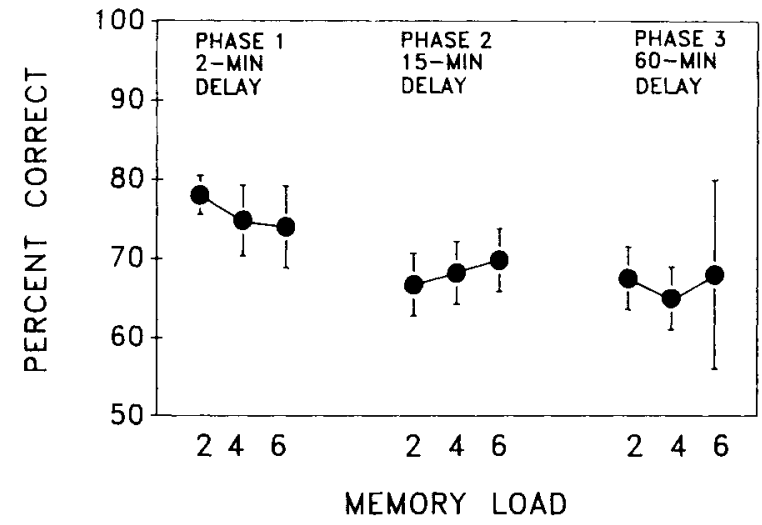

Figure 2. Mean retention accuracy as a function of memory load (two, four, or six sites visited prior to the delay) during the three phases of delay testing in Experiment 2A. Vertical bars indicate standard error of mean. Chance level is $50 \%$.

as has been found in previous studies in an open-field setting (Spetch \& Edwards, 1986; Spetch \& Honig, 1988).

Forced-choice memory load tests. Figure 2 shows mean retention accuracy for the three conditions of working-memory load during the three test phases. The number of sites visited before the delay had no consistent effect on the pigeons' retention accuracy.

One-way analyses of variance on retention accuracy revealed that there was no significant effect of memory load during any of the delay phases (all $F \mathrm{~s}<1$ ). However, retention accuracy was significantly above chance level $(1.0$ correct) at each of the three delays $[2 \mathrm{~min}$, $t(3)=8.64, p<.01 ; 15 \mathrm{~min}, t(3)=13.14, p<.001$; $60 \min , t(3)=6.12, p<.01]$.

Exclusion of trials on which a repeat sample choice was made did not change this pattern of results: analyses of variance still failed to reveal a significant effect of memory load in any of the three phases.

Table 2 shows the retention-accuracy scores for the individual subjects. The scores obtained after excluding trials with repeated choice of a sample are also shown.

\section{Experiment 2B}

\section{Method}

Subjects. The 4 pigeons from Experiment $2 \mathrm{~A}$, together with 2 naive 1 -year-old White King pigeons, served as subjects. They were maintained as described for Experiment $2 \mathrm{~A}$.
Apparatus. The apparatus was the same as that described for Experiment 2A.

Procedure. Pigeons 73, 539, 37, and S84 served in this experiment after completion of Experiment 2A. Pigeons 278 and 275 were first given a few trials of pretraining, followed by 10 trials with a free-choice procedure, as described for Experiment $2 \mathrm{~A}$.

The procedure used during the test phase was identical to that used in Experiment 2A, except that delay was manipulated within a single test phase. Three memory-load conditions (FC-2, FC-4, and FC-6) were tested at each of two delay conditions ( 2 and $60 \mathrm{~min}$ ). Each condition occurred once in each block of six trials. The test phase lasted for a total of 60 trials (10 in each condition).

\section{Results}

During initial free-choice trials, Pigeon 278 made an average of 6.1 correct choices out of 8 and Pigeon 275 made an average of 7.4 correct choices.

Mean retention accuracy is shown as a function of delay for each condition of memory load in Figure 3. Accuracy was consistently lower after a 60-min delay than after a 2-min delay, but the number of sites visited (memory load) did not have a consistent effect. Although accuracy appeared slightly lower in the FC-4 condition than in either FC-2 or FC-6 at the 2-min delay, this pattern was not present at the 60-min delay. Moreover, a two-way analysis of variance revealed a significant effect of delay $[F(1,5)=10.46, p<.05]$, but not of memory load $[F(2,10)=1.07, p>.1]$, and there was no significant interaction between delay and memory load $[F(2,10)=1.31, p>.1]$.

Retention accuracy was significantly above chance level ( $50 \%$ correct) at both the 2 -min delay $[t(5)=7.08, p<$ $.001]$ and the 60 -min delay $[t(5)=4.85, p<.01]$.

Exclusion of data from all trials on which a repeat error was made during the sample period did not alter the pattern of significant and nonsignificant effects. Table 3 shows the retention-accuracy scores for the individual subjects. The scores obtained after excluding trials with repeated choice of a sample are also shown.

\section{Discussion}

The pigeons' accuracy in locating unvisited sites was similar regardless of whether the birds visited two, four, or six different sites prior to the delay. The results do not appear to be contaminated by ceiling or floor effects, because there was room for either increases or decreases

Table 2

Retention-Accuracy Scores for the 4 Subjects in Experiment $2 A$

\begin{tabular}{|c|c|c|c|c|c|c|c|c|c|c|c|c|c|c|c|c|c|c|}
\hline \multirow[b]{4}{*}{ Subject } & \multicolumn{18}{|c|}{ Delay } \\
\hline & \multicolumn{6}{|c|}{$2 \mathrm{~min}$} & \multicolumn{6}{|c|}{$15 \mathrm{~min}$} & \multicolumn{6}{|c|}{$60 \mathrm{~min}$} \\
\hline & \multicolumn{2}{|c|}{ FC-2 } & \multicolumn{2}{|c|}{$\mathrm{FC}-4$} & \multicolumn{2}{|c|}{ FC-6 } & \multicolumn{2}{|c|}{ FC-2 } & \multicolumn{2}{|c|}{ FC-4 } & \multicolumn{2}{|c|}{ FC-6 } & \multicolumn{2}{|c|}{ FC-2 } & \multicolumn{2}{|c|}{$\mathrm{FC}-4$} & \multicolumn{2}{|c|}{ FC-6 } \\
\hline & RE & NRE & $\mathrm{RE}$ & NRE & RE & NRE & $\mathrm{RE}$ & NRE & RE & NRE & $\mathrm{RE}$ & NRE & RE & NRE & RE & NRE & RE & NRE \\
\hline 73 & 1.56 & 1.67 & 1.56 & 1.75 & 1.40 & 1.33 & 1.43 & 1.43 & 1.25 & 1.14 & 1.38 & 1.17 & 1.40 & 1.40 & 1.30 & 1.33 & 0.89 & 0.89 \\
\hline 539 & 1.56 & 1.56 & 1.25 & 1.40 & 1.30 & 1.00 & 1.50 & 1.38 & 1.30 & 1.38 & 1.20 & 1.11 & 1.40 & 1.40 & 1.50 & 1.50 & 1.67 & 1.50 \\
\hline 37 & 1.70 & 1.67 & 1.50 & 1.71 & 1.78 & 2.00 & 1.10 & 1.10 & 1.30 & 1.33 & 1.60 & 1.57 & 1.10 & 1.10 & 1.20 & 1.20 & 1.89 & 1.89 \\
\hline S84 & 1.44 & 1.44 & 1.67 & 1.67 & 1.44 & 1.33 & 1.30 & 1.30 & 1.60 & 1.62 & 1.40 & 1.33 & 1.50 & 1.50 & 1.20 & 1.22 & 1.00 & 1.00 \\
\hline
\end{tabular}

Note-Data represent the number correct out of two choices. FC-2 $=$ forced choice of two randomly selected sites, FC-4 = forced choice of four randomly selected sites, FC-6 $=$ forced choice of six randomly selected sites, RE $=$ retention-accuracy scores with repeat errors during the sample phase included, NRE = retention-accuracy scores with repeat errors during the sample phase excluded. 
Table 3

Retention-Accuracy Scores for the 6 Subjects in Experiment 2B

\begin{tabular}{|c|c|c|c|c|c|c|c|c|c|c|c|c|}
\hline \multirow[b]{4}{*}{ Subject } & \multicolumn{12}{|c|}{ Delay } \\
\hline & \multicolumn{6}{|c|}{$2 \mathrm{~min}$} & \multicolumn{6}{|c|}{$60 \mathrm{~min}$} \\
\hline & \multicolumn{2}{|c|}{ FC-2 } & \multicolumn{2}{|c|}{ FC-4 } & \multicolumn{2}{|c|}{ FC- 6} & \multicolumn{2}{|c|}{ FC-2 } & \multicolumn{2}{|c|}{ FC-4 } & \multicolumn{2}{|c|}{ FC-6 } \\
\hline & RE & NRE & $\mathrm{RE}$ & NRE & RE & NRE & $\overline{R E}$ & NRE & RE & NRE & RE & NRE \\
\hline 73 & 2.00 & 2.00 & 1.89 & 1.89 & 1.89 & 1.89 & 1.33 & 1.33 & 1.67 & 1.75 & 2.00 & 1.89 \\
\hline 539 & 1.56 & 1.56 & 1.56 & 1.44 & 1.67 & 1.67 & 1.44 & 1.56 & 1.67 & 1.56 & 1.56 & 1.44 \\
\hline 37 & 2.00 & 2.00 & 1.89 & 1.89 & 1.89 & 1.89 & 1.33 & 1.38 & 1.11 & 1.11 & 1.44 & 1.50 \\
\hline S84 & 1.78 & 1.88 & 1.67 & 1.75 & 1.78 & 1.67 & 1.33 & 1.25 & 1.33 & 1.17 & 1.44 & 1.29 \\
\hline 278 & 1.50 & 1.44 & 1.00 & 0.71 & 1.40 & 1.33 & 1.20 & 1.11 & 1.50 & 1.56 & 1.00 & 1.00 \\
\hline 275 & 1.67 & 1.67 & 1.44 & 1.43 & 1.78 & 1.80 & 1.33 & 1.33 & 1.22 & 1.12 & 1.00 & 0.83 \\
\hline
\end{tabular}

Note-Data represent the number correct out of two choices. FC-2 $=$ forced choice of two randomly selected sites, FC- $4=$ forced choice of four randomly selected sites, FC- $6=$ forced choice of six randomly selected sites, RE = retention-accuracy scores with repeat errors during the sample phase included, NRE = retentionaccuracy scores with repeat errors during the sample phase excluded.

in performance at both delays. Thus, working-memory load within the range tested does not seem to be an important determinant of pigeons' spatial memory performance in the open-field setting. This claim, of course, rests on the assumption that accurate performance in the present open-field task does reflect spatial memory processes.

\section{EXPERIMENT 3}

The relevance of the results of Experiments 1 and 2 to the understanding of spatial memory processes in pigeons hinges on the assumption that accurate performance in the present open-field task reflects spatial memory, rather than memory for specific cartons visited regardless of their spatial location. Spetch and Edwards (1986) provided evidence that pigeons do not need carton-specific cues to avoid revisiting sites: in that study, pigeons continued to choose accurately when all sites were identical. However, additional evidence showing that pigeons remember the spatial location they have visited would be useful, because the tests conducted by Spetch and Edwards did not use a forced-choice procedure and the setting and cartons they used were not identical to those used in the present experiments. Thus, one purpose of Experiment 3 was to provide further evidence that pigeons use spatial cues to solve the open-field task.

A second purpose of Experiment 3 was to examine the relative control of pigeons' working memory by the various redundant but relevant cues that had been provided in the open-field task used in the first two experiments. Spetch and Edwards (1986) showed that carton-specific cues are not necessary for accurate performance, but their results did not indicate the degree to which these cues might be used when available. Indeed, studies with both pigeons (Spetch \& Edwards, 1988) and rats (Kraemer, Gilbert, \& Innis, 1983) have indicated that these animals exhibit a flexible use of redundant sources of information in spatial memory tasks. In Experiment 3, several sets of potential cues were manipulated during the delay interval of test trials: the cartons, floor objects, wall pictures, and the noise source. To the extent that these cues were used by the pigeons to distinguish visited from unvisited sites, accuracy (defined in terms of the correct location in the room) should be reduced on these tests.

\section{Method}

Subjects. Pigeons 73, 539, 37, and S84 served in this experiment following completion of Experiment 2B. Housing and feeding conditions were as described in Experiment $2 \mathrm{~A}$.

Apparatus. The apparatus was the same as that described for Experiment $2 \mathrm{~A}$.

Procedure. All trials used the FC-2 procedure described in Experiments $2 \mathrm{~A}$ and $2 \mathrm{~B}$ (i.e., two cartons were available during the sample period and four were available during the test period). The delay interval between the sample period and the test was $10 \mathrm{~min}$ throughout this experiment. Five types of test trials were conducted in which specific cues were manipulated: (1) carton reversal, (2) object rotation, (3) picture rotation, (4) noise rotation, and (5) cartons, objects, and pictures. Each test block of trials contained one test trial of each type, preceded by one control trial. The order of the five types of tests varied across test blocks. Between test blocks, the pigeons received between 5 and 10 baseline trials to maintain or reestablish high levels of baseline accuracy. Testing continued until each bird had received five test trials of each type.

For the carton-reversal tests, two randomly selected cartons were present during the sample part of the trial. During the delay, two additional randomly selected cartons were added to the room, and the sample cartons and the new ones were then interchanged so that the correct (i.e., unvisited) location in the room contained a carton that had been previously visited. Thus, carton-specific cues were placed in opposition with all other cues. To prevent reinforcement contingencies during the tests from biasing the pigeons toward the use of particular cues, all four cartons were baited for the retention test, but the birds were permitted only two choices. Accuracy was scored in terms of the correct location in the room. That is, choice of a previously visited room location (even though it now contained an unvisited carton) was scored as an error. Control trials, which preceded the carton-reversal tests, were identical except that the cartons were not interchanged during the delay, and only the two unvisited sites were baited.

For the four remaining types of tests, as well as for the control trials that preceded them, the two sample sites and the two additional choice sites were selected to be nonoverlapping sets. That is, for each trial, two sample sites were randomly selected, with the constraint that they be either adjacent to or separated from one another by no more than two locations. The two additional choice sites were then randomly selected, with the constraint that neither site was adjacent to a sample site. These constraints resulted in the 


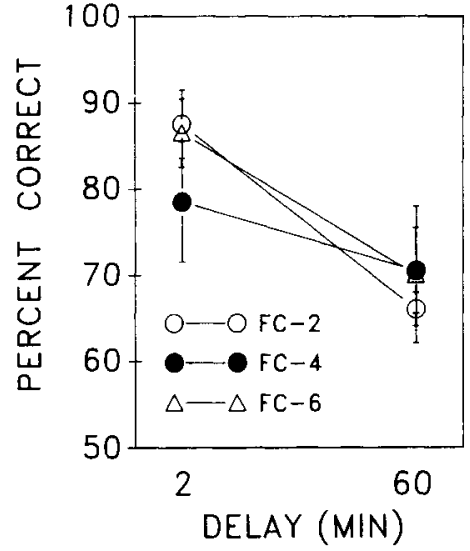

Figure 3. Mean retention accuracy as a function of delay for each of the three conditions of memory load (two, four, or six sites visited prior to the delay) in Experiment 2B. Vertical bars indicate standard error of mean. Chance level is $\mathbf{5 0} \%$.

location of the sets of correct and incorrect sites in different hemicircles and meant that a $180^{\circ}$ rotation of the objects, pictures, or noise source would tend to place these cues in opposition with the remaining cues. For the object-rotation, picture-rotation, and noiserotation tests, the four floor objects, the four wall pictures, or the noise source, respectively, were rotated by $180^{\circ}$ during the delay. For the cartons, objects, and pictures tests, the visited cartons were interchanged with the unvisited ones, and the objects and pictures were also rotated by $180^{\circ}$. Accuracy was scored in terms of the correct room location, and all cartons were baited during test trials.
All other aspects of the procedure were as described in Experiment $2 \mathrm{~A}$.

\section{Results}

Figure 4 shows mean accuracy (percentage of choices to the correct room location) during the carton-reversal, object-rotation, picture-rotation, and noise-rotation tests, and during the control trials that preceded each of these tests. Accuracy in selecting the correct room location remained high during all of these tests, and in none of these cases was accuracy on test trials significantly different from accuracy on the preceding control trials (all $t \mathrm{~s}<1.7)$. Figure 5 shows the results of the tests in which the cartons, objects, and pictures were manipulated together. In this case, accuracy was significantly lower on test trials than on the preceding baseline trials $[t(3)=$ $4.37, p<.05]$.

Table 4 shows the average scores for each of the 4 subjects on the five types of tests and their preceding control trials.

\section{Discussion}

The pigeons showed a clear preference for roomlocation cues over carton-specific cues. No significant effect on the pigeons' choice behavior was observed when the cartons, objects, pictures, or noise source were manipulated individually, which indicates that none of these cues served as a dominant source of information.
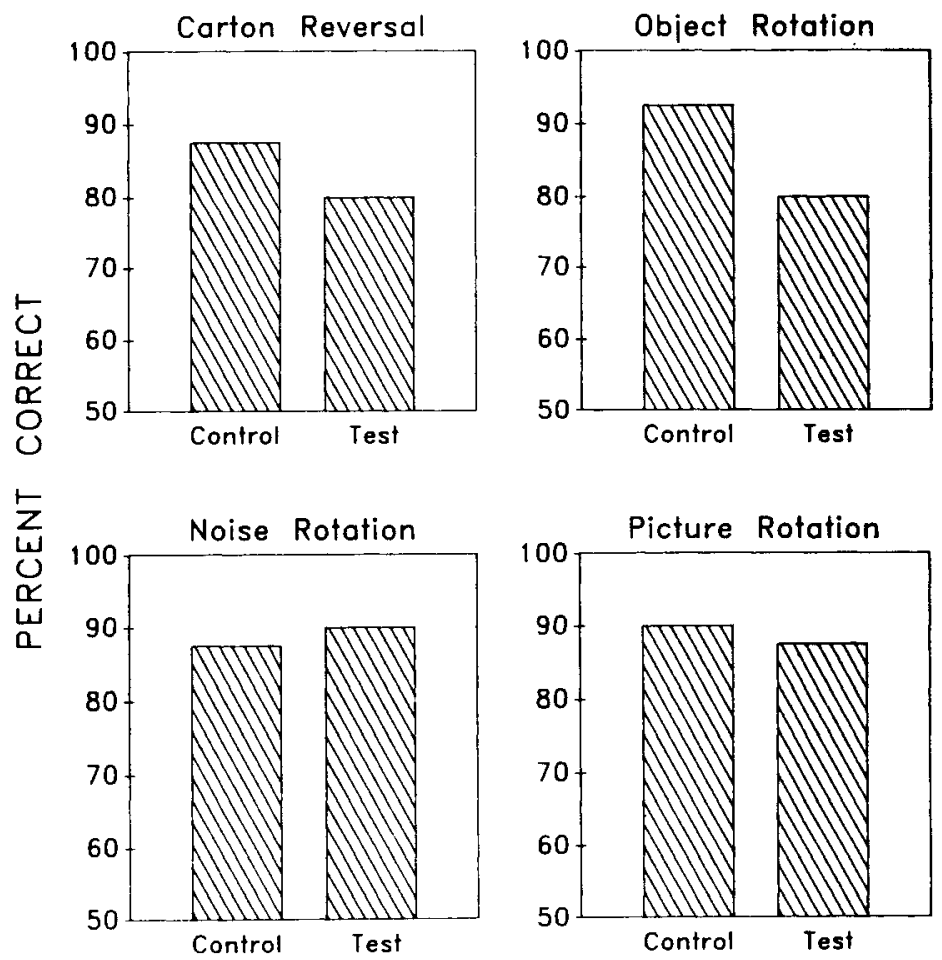

Figure 4. Mean retention accuracy (choice of sites in correct room location) during test trials in which the cartons were reversed, the objects were rotated, the noise source was rotated, or the pictures were rotated during the delay interval and during the control trials that preceded each of these tests. 
Cartons, Objects, \& Pictures

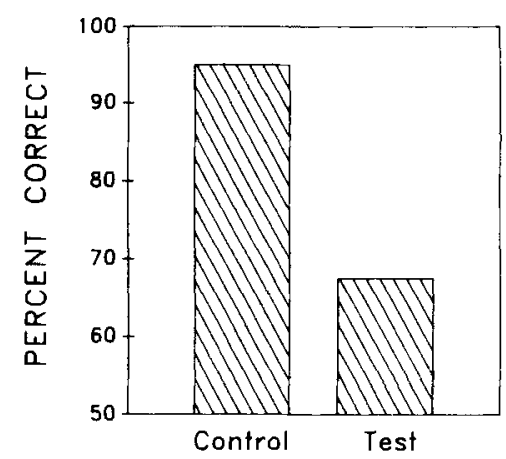

Figure 5. Mean retention accuracy (choice of sites in correct room location) during test trials in which the cartons were reversed and the objects and pictures were rotated during the delay interval and during the control trials that preceded these tests.

However, concurrent manipulation of the cartons, objects, and pictures did disrupt accuracy, which indicates that these cues exerted some control. Finally, because all cartons were baited during the tests, the high level of accuracy observed on four of the tests clearly rules out the possibility that the pigeons relied on cues emanating from the food itself to distinguish between visited and unvisited sites.

\section{GENERAL DISCUSSION}

Between-experiment comparisons of pigeons' retention abilities in a T-maze (Olson \& Maki, 1983), a four-arm maze (Dale, 1988), and an eight-arm maze (Roberts \& Van Veldhuizen, 1985) had suggested that pigeons' ability to retain spatial information over a delay might depend on working-memory load (Dale, 1988; Roberts \& Van Veldhuizen, 1985). The present results failed to support this suggestion: in Experiments 1 and 2, the pigeons' retention accuracy in the open-field task declined as a function of delay, but the rate of this decline was not affected by the number of sites visited (2-6) prior to the delay interval. Although increases in memory load beyond the range tested here may affect pigeons' spatial retention, the range used was similar to that previously hy- pothesized to affect pigeons' retention in spatial memory tasks (Dale, 1988; Roberts \& Van Veldhuizen, 1985).

The failure to observe a significant effect of workingmemory load is surprising, not only because of suggestions in the literature that it might be an important variable, but also because one would expect that potential confusion between the sites in working memory would be greater in the large memory-load conditions than in the small memory-load conditions. For example, in the FC- 6 condition, each of the two correct sites would always be adjacent to one of the six previously visited locations (although not necessarily to the ones present during the test). In contrast, in the FC-2 condition, the correct sites would only sometimes be adjacent to a visited site. Thus, it is interesting that the pigeons did not make more confusion errors after having visited several of the sites.

In the two experiments entailing within-phase manipulations of the delay interval, the pigeons showed a significant reduction in accuracy at a 1 -h delay relative to a 15-min delay (Experiment 1) or a 2-min delay (Experiment $2 \mathrm{~B}$ ), although accuracy was still significantly above chance level at the 1-h delay. These results are consistent with those obtained in a previous experiment using an open-field task: Spetch and Honig (1988) found that pigeons' accuracy remained high across delays of 2$32 \mathrm{~min}$, but declined to near chance level at a 2-h delay. Thus, in this open-field task, pigeons' accuracy appears to decline with delays of $1 \mathrm{~h}$ or more. This delay is somewhat shorter than the delay at which rats (Beatty \& Shavalia, 1980) or Clark's nutcrackers (Balda \& Kamil, 1988) begin to show significant forgetting. Although pigeons' ability to retain spatial information over delays does not appear to be determined by working-memory load, it is possible that the number of sites in the reference-memory set, or the number of sites present at the time of choice, are important variables.

Experiment 3 confirmed that retention of spatial information controls performance in the present open-field task. The pigeons avoided the place in the room that they had previously visited, rather than the specific carton they had visited. This finding extends previous evidence from an open-field task that indicated that pigeons do not rely on carton-specific cues for working memory (Spetch \& Edwards, 1986), and is consistent with evidence from

Table 4

Mean Retention Accuracy for Cue-Manipulation Tests and Their Preceding Control Trials in Experiment 3

\begin{tabular}{|c|c|c|c|c|c|c|c|c|}
\hline \multirow[b]{3}{*}{ Cue Manipulated } & \multicolumn{8}{|c|}{ Subject } \\
\hline & \multicolumn{2}{|c|}{ Bird 73} & \multicolumn{2}{|c|}{ Bird 539} & \multicolumn{2}{|c|}{ Bird 37} & \multicolumn{2}{|c|}{ Bird S84 } \\
\hline & Control & Test & Control & Test & Control & Test & Control & Test \\
\hline Cartons & 1.8 & 1.4 & 1.4 & 1.6 & 2.0 & 1.6 & 1.8 & 1.8 \\
\hline Objects & 2.0 & 1.6 & 1.8 & 1.2 & 2.0 & 2.0 & 1.6 & 1.6 \\
\hline Pictures & 1.8 & 2.0 & 1.8 & 1.8 & 1.8 & 1.6 & 1.8 & 1.6 \\
\hline Noise & 2.0 & 2.0 & 1.6 & 1.8 & 1.8 & 2.0 & 1.6 & 1.8 \\
\hline $\begin{array}{l}\text { Cartons, Objects, } \\
\text { and Pictures }\end{array}$ & 1.8 & 1.2 & 1.8 & 1.0 & 2.0 & 1.4 & 2.0 & 1.8 \\
\hline
\end{tabular}

Note-Data represent the number correct out of two choices. 
radial-arm maze studies indicating that extramaze cues tend to be dominant for pigeons (Roberts \& Van Veldhuizen, 1986) as well as for rats (e.g., Kraemer et al., 1983), even when distinctive intramaze cues are available. The present finding also seems consistent with some recent work by Sherry (1989) on the cues chickadees use to relocate their food caches. In that study, chickadees stored food in an arena containing a number of storage sites on branches of trees. Next to each storage site was a colored card that provided a local cue. On each wall of the arena was a large picture that provided a distal cue. Manipulation of these cues between the caching and retrieval periods indicated that the chickadees relied primarily on the distal cues rather than on the local cues adjacent to each site.

The cue-manipulation tests of Experiment 3 indicated that the location in the room exerted primary control of the pigeons' choice behavior. Cues provided by the cartons, objects, pictures, and noise source exerted little control when manipulated individually, although concurrent manipulation of the cartons, objects, and pictures had a significant effect on choice behavior. At first glance, these results seem somewhat inconsistent with results obtained by Spetch and Honig (1988), who found that manipulation of both floor objects and wall pictures between trials disrupted spatial working memory. On the basis of these results, it seems surprising that the within-trial manipulation of objects or pictures did not by themselves produce a significant disruption. However, it should be noted that the concurrent manipulation of these cues, which did produce a significant disruption in accuracy, would be most comparable to the manipulation in the Spetch and Honig study.

The significant disruption in accuracy produced by the concurrent manipulation of the cartons, pictures, and objects, but not by manipulation of any of these cues alone, suggests that pigeons' choices might be controlled by a weighted average of information provided by several cues (see Cheng, 1989a, 1989b). When only one cue is shifted, the average information may not change sufficiently to alter choice behavior in the present task, particularly if the shifted cue carries a low weighting. But a concurrent shift in several cues, each of which has a low weighting, might produce a substantial enough change in average information to alter choice behavior.

\section{REFERENCES}

BALDA, R. P., \& KAMIL, A. C. (1988). The spatial memory of Clark's nutcrackers (Nucifraga columbiana) in an analogue of the radial arm maze. Animal Learning \& Behavior, 16, 116-122.
Beatty, W. W., \& Shavalia, D. A. (1980). Spatial memory in rats: Time course of working memory and effects of anesthetics. Behavioral \& Neural Biology. 28, 454-462.

Bond, A. B., CooK, R. G., \& LAMB, M. R. (1981). Spatial memory and the performance of rats and pigeons in the radial-arm maze. Animal Learning \& Behavior, 9, 575-580.

ChEng, K. (1989a, June). Three psychophysical principles in the processing of spatial and temporal information. Paper presented at the conference on Cognitive Aspects of Stimulus Control, Dalhousie University, Halifax, Nova Scotia.

CHENG, K. (1989b). The vector sum model of pigeon landmark use. Journal of Experimental Psychology: Animal Behavior Processes, 15, 366-375.

Cook, R. G., Brown, M. F., \& Riley, D. A. (1985). Flexible memory processing by rats: Use of prospective and retrospective information in the radial maze. Journal of Experimental Psychology: Animal Behavior Processes, 11, 453-469.

DALE, R. H. I. (1988). Spatial memory in pigeons on a four-arm radial maze. Canadian Journal of Psychology, 42, 78-83.

Honig, W. K. (1978). Studies of working memory in the pigeon. In S. Hulse, H. Fowler, \& W. K. Honig (Eds.), Cognitive processes in animal behavior (pp. 211-248). Hillsdale, NJ: Erlbaum.

Kraemer, P. J., Gilbert, M. E., \& InNIS, N. K. (1983). The influence of cue type and configuration upon radial-maze performance in the rat. Animal Learning \& Behavior, 11, 373-380.

OLSON, D. J., \& MAKI, W. S. (1983). Characteristics of spatial memory in pigeons. Journal of Experimental Psychology: Animal Behavior Processes, 9, 266-280.

RoberTs, W. A., \& DALE, R. H. I. (1981). Remembrance of places lasts: Proactive inhibition and patterns of choice in rat spatial memory. Learning \& Motivation, 12, 261-281.

Roberts, W. A., \& VAN Veldhuizen, N. (1985). Spatial memory in pigeons on the radial maze. Journal of Experimental Psychology: Animal Behavior Processes, 11, 241-260.

SHERRY, D. F. (1989, June). Landmarks, the hippocampus, and spatial search in food-storing birds. Paper presented at the conference on Cognitive Aspects of Stimulus Control, Dalhousie University, Halifax, Nova Scotia.

SPETCH, M. L., \& EDWARDS, C. A. (1986). Spatial memory in pigeons (Columba livia) in an open-field feeding environment. Joumal of Comparative Psychology, 100, 266-278.

SPETCH, M. L., \& Edwards, C. A. (1988). Pigeons' (Columba livia) use of global and local cues for spatial memory. Animal Behaviour, 36, 293-295.

SPETCH, M. L., \& HoNIG, W. K. (1988). Characteristics of pigeons' spatial working memory in an open-field task. Animal Learning \& Behavior, 16, 123-131.

Zentall, T. R., Steirn, J. N., \& Jackson-Smith, P. (1987, November). Spatial learning in the pigeon: An operant analog of the radialarm maze. Paper presented at the 28th Annual Meeting of the Psychonomic Society, Seattle, WA. (Abstract: Bulletin of the Psychonomic Society, 25, 341).
(Manuscript received May 9, 1989; revision accepted for publication January 8,1990 .) 